PROCEEDINGS OF THE

AMERICAN MATHEMATICAL SOCIETY

Volume 137, Number 1, January 2009, Pages 1-9

S 0002-9939(08)09607-X

Article electronically published on July 18, 2008

\title{
INNER IDEAL STRUCTURE OF NEARLY ARTINIAN LIE ALGEBRAS
}

\author{
ANTONIO FERNÁNDEZ LÓPEZ, ESTHER GARCÍA, AND MIGUEL GÓMEZ LOZANO
}

(Communicated by Gail R. Letzter)

\begin{abstract}
In this paper we study the inner ideal structure of nondegenerate Lie algebras with essential socle, and characterize, in terms of the whole algebra, conditions under which the socle is Artinian.
\end{abstract}

\section{INTRODUCTION}

Let $L$ be a Lie algebra over a ring of scalars $\Phi$. A $\Phi$-submodule $B$ of $L$ is an inner ideal if $[B,[B, L]] \subset B$, and $B$ is abelian if $[B, B]=0$. The initial motivation to study inner ideals in Lie algebras was due to the fact that inner ideals are closely related to ad-nilpotent elements, and certain restrictions on these elements yield an elementary criterion for distinguishing the nonclassical from the classical (finite dimensional) simple Lie algebras over algebraically closed fields of characteristic greater than 5 [2].

In 1, G. Benkart examined the Lie inner ideal structure of semiprime associative rings, and of the skew elements of prime rings with involution. An extension of these results was carried out by the authors in [6], where the inner ideals of infinite dimensional finitary simple Lie algebras were described.

Inner ideals have also become a key notion in developing a socle theory for nondegenerate Lie algebras [4, and were used in [9] to construct gradings of Lie algebras. This requires the existence of abelian inner ideals whose subquotient, a Jordan pair, is covered by a finite grid, and it produces a grading of the Lie algebra by the weight lattice of the root system associated to the covering grid.

Very recently [8, inner ideals, and their associated notions of kernel and complement, have allowed us to obtain a Lie algebra analogue of the module theoretic characterization of semiprime one-sided Artinian associative rings ( $R$ is unital and completely reducible as a module), which parallels that due to O. Loos and E. Neher for Jordan systems [13.

Received by the editors November 14, 2007.

2000 Mathematics Subject Classification. Primary 17B05; Secondary 17 B60.

Key words and phrases. Artinian Lie algebras, inner ideals, socle, complementation.

The first author was partially supported by the MEC and Fondos FEDER, MTM2007-61978.

The second author was partially supported by the MEC and Fondos FEDER, MTM200406580-C02-01 and MTM2007-62390, by the Plan de Investigación del Principado de Asturias FICYT-IB05-017, and by the Programa de Creación y Consolidación de Grupos de Investigación URCJ-CM-2006-CET-0585.

The third author was partially supported by the MEC and Fondos FEDER, MTM2007-61978, and by the Plan de Investigación del Principado de Asturias FICYT-IB05-017. 
Any nondegenerate Artinian Jordan pair agrees with its socle [12. However, as mentioned in [8, there are examples of nondegenerate Artinian Lie algebras which do not coincide with their socles, although any nondegenerate Artinian Lie algebra has an essential Artinian socle 44. In this paper we look into the inner ideal structure of nondegenerate Lie algebras with essential socle, and study what "having an essential Artinian socle" means for the whole algebra. This property will be related to being complemented (the socle) or abelian complemented (the whole algebra). Indeed, we show the following result:

Theorem. For a nondegenerate Lie algebra $L$ over a ring of scalars $\Phi$ in which 2, 3, 5,7 are invertible with essential socle $S$, the following conditions are equivalent:

(i) $S$ is Artinian.

(ii) $S$ is a complemented Lie algebra and has finitely many ideals.

(iii) $L$ is abelian complemented and has finitely many simple ideals.

\section{LIE ALGEBRAS AND JORDAN PAIRS}

1.1. Throughout this paper, and unless otherwise specified, we will be dealing with Lie algebras $L$ [10, [14] (with $[x, y]$ denoting the Lie bracket and $\operatorname{ad}_{x}$ the adjoint map determined by $x$ ) and Jordan pairs $V=\left(V^{+}, V^{-}\right)$[1] (with Jordan triple products $\{x, y, z\}$, for $x, z \in V^{\sigma}, y \in V^{-\sigma}, \sigma= \pm$ ) over a ring of scalars $\Phi$ containing $\frac{1}{6}$. So the Jordan pairs considered here are linear and the quadratic maps are $Q_{x} y=\frac{1}{2}\{x, y, x\}$.

1.2. Let $V=\left(V^{+}, V^{-}\right)$be a Jordan pair. An element $x \in V^{\sigma}, \sigma= \pm$, is called an absolute zero divisor if $Q_{x}=0$. Thus $V$ is said to be nondegenerate if it has no nonzero absolute zero divisors, semiprime if $Q_{B^{ \pm}} B^{\mp}=0$ implies $B=0$, and prime if $Q_{B^{ \pm}} C^{\mp}=0$ implies $B=0$ or $C=0$, for any ideals $B=\left(B^{+}, B^{-}\right), C=\left(C^{+}, C^{-}\right)$ of $V$. Similarly, given a Lie algebra $L, x \in L$ is an absolute zero divisor of $L$ if $\operatorname{ad}_{x}^{2}=0 ; L$ is nondegenerate if it has no nonzero absolute zero divisors, semiprime if $[I, I]=0$ implies $I=0$, and prime if $[I, J]=0$ implies $I=0$ or $J=0$, for any ideals $I, J$ of $L$. A Jordan pair or Lie algebra is strongly prime if it is prime and nondegenerate. We note that any ideal of a nondegenerate Lie algebra is again nondegenerate; see [16, Lemma 4]. A Lie algebra is simple if it is nonabelian and contains no proper ideals.

1.3. Given a Jordan pair $V=\left(V^{+}, V^{-}\right)$, an inner ideal of $V$ is any $\Phi$-submodule $I$ of $V^{\sigma}$ such that $\left\{I, V^{-\sigma}, I\right\} \subset I$. Similarly, an inner ideal of a Lie algebra $L$ is a $\Phi$-submodule $B$ of $L$ such that $[B,[B, L]] \subset B$. An abelian inner ideal is an inner ideal $B$ which is also an abelian subalgebra, i.e., $[B, B]=0$.

1.4. The annihilator of an ideal $I$ in a Lie algebra $L$ is defined as Ann $I=\{x \in$ $L \mid[x, I]=0\}$. If $L$ is nondegenerate, then Ann $I=\{x \in L \mid[x,[I, x]]=0\}$ and $I \cap$ Ann $I=0$ [5, 2.5]. In this case, essential ideals of $L$ have zero annihilator and hit nonzero inner ideals: if $I$ is an essential ideal of $L$ and $B$ is an inner ideal of $L$, then $[B,[B, I]] \subset B \cap I=0$ would imply $B \subset$ Ann $I=0$.

1.5. An ad-nilpotent element $x \in L$ of index of nilpotency $\leq 3$ is called a Jordan element. In this case, $\operatorname{ad}_{x}^{2} a$ is also a Jordan element for any $a \in L[7,2.3$ (viii)]. By [2. 1.7(iii)], any Jordan element $x \in L$ satisfies the following analogue of the Jordan identity:

$$
\operatorname{ad}_{\mathrm{ad}_{x}^{2} y}^{2}=\operatorname{ad}_{x}^{2} \operatorname{ad}_{y}^{2} \operatorname{ad}_{x}^{2}
$$


for any $y \in L$. Clearly, any element of an abelian inner ideal is a Jordan element. Conversely, by [2, 1.8], any Jordan element $b \in L$ yields the abelian inner ideals $[b]:=[b,[b, L]]$ and $(b):=\Phi b+[b]$.

Proposition 1.6. Let $L$ be a nondegenerate Lie algebra $\left(\frac{1}{6} \in \Phi\right)$, let $I$ be an ideal of $L$, and let $x \in I$ be a Jordan element of $I$. Then $x$ is a Jordan element of $L$.

Proof. Since $\operatorname{ad}_{x}^{3} I=0$ and $I$ is an ideal of $L, \operatorname{ad}_{x}^{4} L=0$. So, for every $a \in L$,

$$
\begin{aligned}
0 & =\operatorname{ad}_{\mathrm{ad}_{x}^{4} a}=X^{4} A-4 X^{3} A X+6 X^{2} A X^{2}-4 X A X^{3}+A X^{4} \\
& =-4 X^{3} A X+6 X^{2} A X^{2}-4 X A X^{3}
\end{aligned}
$$

where capital letters denote the adjoint maps with respect to those elements. Since $\operatorname{ad}_{x}^{2}[x, a]$ is a Jordan element of $I$, for every $y \in I$ we have

$$
\begin{aligned}
\operatorname{ad}_{\mathrm{ad}_{x}^{2}[x, a]}^{2} y & =\operatorname{ad}_{x}^{2} \operatorname{ad}_{[x, a]}^{2} \operatorname{ad}_{x}^{2} y=\left(X^{2}(X A-A X)^{2} X^{2}\right) y \\
& =\left(-X^{2} A X^{2} A X^{2}+X^{2} A X A X^{3}\right) y=-X^{2} A X^{2} A X^{2} y \\
& =\left(-\frac{2}{3} X^{2} A X^{3} A X-\frac{2}{3} X^{2} A X A X^{3}\right) y=0 \text { (by (1)). }
\end{aligned}
$$

$\operatorname{Then}^{2} \operatorname{ad}_{x}^{2}[x, a] \in I \cap \operatorname{Ann} I=0$, so $x$ is a Jordan element of $L$.

1.7. The socle of a nondegenerate Lie algebra $L$ is defined as the sum of all minimal inner ideals of $L$. By [4, Theorem 2.5], Soc $L$ is an ideal of $L$ and a direct sum $\bigoplus_{\alpha} M_{\alpha}$ of simple ideals $M_{\alpha}$ of $L$. Moreover, each simple component $M_{\alpha}$ of Soc $L$ is either inner simple or contains an abelian minimal inner ideal [2, Theorem 1.12].

A Lie algebra $L$ is said to be Artinian if it satisfies the descending chain condition on all inner ideals. Simple nondegenerate Artinian Lie algebras coincide with their socles.

1.8. A Jordan element $e \in L$ is called von Neumann regular if $e \in \operatorname{ad}_{e}^{2} L$. Assume that $\frac{1}{30} \in \Phi$. Then

(i) Any von Neumann regular element $e$ of $L$ can be extended to an idempotent $(e, f)$ (see [15, V.8.2] or [4, Proposition 1.18]), i.e.,

$$
\operatorname{ad}_{e}^{3}=\operatorname{ad}_{f}^{3}=0,[[e, f], e]=2 e \text { and }[[e, f], f]=-2 f .
$$

Note that the last two conditions imply that $(e,[e, f], f)$ is a $\mathfrak{s l}(2)$-triple.

(ii) Any idempotent $(e, f)$ yields a 5-grading $L=L_{-2} \oplus L_{-1} \oplus L_{0} \oplus L_{1} \oplus L_{2}$ (called the Peirce decomposition of $(e, f)$ ), where each $L_{i}$ is the eigenspace of the adsemisimple element $h:=[e, f]$ relative to the eigenvalue $i, i=0, \pm 1, \pm 2$. Moreover, $L_{2}=[e]$ and $L_{-2}=[f] ;$ see [4, 1.18].

Proposition 1.9. Let $L$ be a nondegenerate Lie algebra $\left(\frac{1}{30} \in \Phi\right)$, let $I$ be an ideal of $L$, and let $B$ be an abelian inner ideal of $I$. If any element of $B$ is von Neumann regular, then $B$ is an inner ideal of $L$.

Proof. Since $B$ is an abelian inner ideal of $I$, any $x \in B$ is a Jordan element of $I$ and hence, by Proposition [1.6, a Jordan element of $L$. Let $y \in I$ be such that $x=\operatorname{ad}_{x}^{2} y$. By 1.5. $\operatorname{ad}_{x}^{2} L=\operatorname{ad}_{\operatorname{ad}_{x}^{2} y}^{2} L=\operatorname{ad}_{x}^{2} \operatorname{ad}_{y}^{2} \operatorname{ad}_{x}^{2} L \subset \operatorname{ad}_{x}^{2} I \subset B$.

1.10. Let $M$ be an inner ideal of a Lie algebra $L$. The kernel of $M$ is the set Ker $M=\{x \in L:[M,[M, x]]=0\}$. For any abelian inner ideal $M$ of $L$, the pair of 
$\Phi$-modules $V=(M, L / \operatorname{Ker} M)$ with triple products given by

$$
\begin{aligned}
& \{m, \bar{a}, n\}:=[[m, a], n] \quad \text { for every } m, n \in M \text { and } a \in L, \\
& \{\bar{a}, m, \bar{b}\}:=\overline{[[a, m], b]} \quad \text { for every } m \in M \text { and } a, b \in L,
\end{aligned}
$$

where $\bar{x}$ denotes the coset of $x$ relative to the submodule $\operatorname{Ker} M$, is a Jordan pair, called the subquotient of $L$ with respect to $M$ [9, Lemma 3.2].

1.11. A complement of an inner ideal $M$ of $L$ is another inner ideal $N$ of $L$ such that $L=M \oplus \operatorname{Ker} N=N \oplus \operatorname{Ker} M$. A Lie algebra $L$ is said to be (abelian) complemented if any (abelian) inner ideal $M$ of $L$ has an (abelian) complement. It was shown in [8, 3.7(iii)] that complemented Lie algebras are abelian complemented. Moreover, subquotients of abelian complemented Lie algebras are complemented as Jordan pairs [8, 3.4].

\section{Lie Algebras With ESSENTIAL SOCle}

Lemma 2.1. Let $L$ be a Lie algebra $\left(\frac{1}{30} \in \Phi\right)$, let $(e, f)$ be a nontrivial idempotent of $L$ with Peirce decomposition $L=L_{-2} \oplus L_{-1} \oplus L_{0} \oplus L_{1} \oplus L_{2}$, and let $I$ be an abelian inner ideal of $L$ such that $e \in I$. Then:

(i) $I=I_{0}+I_{1}+I_{2}$, with $I_{i}=L_{i} \cap I$ for each index $i$, and $I_{0}, I_{2}$ are inner ideals of $L$.

(ii) If $0 \neq y_{0} \in I_{0}$ is von Neumann regular, then so is $e^{\prime}=y_{0}+e$, and both $e, y_{0} \in\left[e^{\prime}\right]$.

(iii) If $L$ is nondegenerate and $I_{0}=0$, then $I_{1}$ is also an inner ideal of $L$.

Proof. (i) Since $e \in[e]=L_{2} \subset I$, for any $y=y_{-2}+y_{-1}+y_{0}+y_{1}+y_{2} \in I$ we have $0=[y, e]=\left[y_{-2}, e\right]+\left[y_{-1}, e\right]+\left[y_{0}, e\right] \in L_{0} \oplus L_{1} \oplus L_{2}$. Hence

$$
\left[y_{i}, e\right]=0, \text { for } i=-2,-1,0,1,2 \text {. }
$$

Then

$$
\begin{aligned}
& y_{-2}=-\frac{1}{2}\left[h, y_{-2}\right]=-\frac{1}{2}\left[[e, f], y_{-2}\right]=-\frac{1}{2}\left[\left[e, y_{-2}\right], f\right]=0, \\
& y_{-1}=-\left[h, y_{-1}\right]=-\left[[e, f], y_{-1}\right]=-\left[\left[e, y_{-1}\right], f\right]=0 .
\end{aligned}
$$

Therefore, $I \subset L_{0} \oplus L_{1} \oplus L_{2}$. Now $y_{1}+2 y_{2}=[h, y]=[[e, f], y] \in I$. Hence $y_{1}=[h, y]-2 y_{2} \in I$ and $y_{0}=y-y_{1}-y_{2} \in I$. Thus $I=I_{0} \oplus I_{1} \oplus I_{2}$, with $I_{i}=I \cap L_{i}$.

Clearly, $I_{2}=L_{2}$ is an inner ideal (since $[e]=L_{2} \subset I$ ); let us now show that $I_{0}$ is also an inner ideal of $L$. By $(1),\left[\left[y_{0}, f\right], e\right]=\left[\left[y_{0}, e\right], f\right]-\left[y_{0}, h\right]=0$, so $0=\left[\left[\left[y_{0}, f\right], e\right], f\right]=\left[\left[y_{0}, f\right], h\right]=-2\left[y_{0}, f\right]$ and hence

$$
\left[y_{0}, f\right]=0 .
$$

Now, given $y_{0} \in I_{0}$, we have

$$
\begin{gathered}
{\left[y_{0}, L_{2}\right] \subset[I, I]=0 \text { (because } I \text { is abelian), }} \\
{\left[y_{0}, L_{-2}\right]=\left[y_{0},\left[f,\left[f, L_{2}\right]\right]\right]=\left[f,\left[f,\left[y_{0}, L_{2}\right]\right]\right]=0(\text { by }(2)),}
\end{gathered}
$$

$\left[y_{0},\left[y_{0}, L_{-1}\right]\right] \subset L_{-1} \cap I=L_{-1} \cap\left(I_{0} \oplus I_{1} \oplus I_{2}\right)=0$, and $\left[y_{0},\left[y_{0}, L_{1}\right]\right]=\left[y_{0},\left[y_{0},\left[e, L_{-1}\right]\right]\right]$ $=\left[e,\left[y_{0},\left[y_{0}, L_{-1}\right]\right]\right]=0$ (by (1) and the fact, proved in [2, 2.1], that $\left.L_{1}=\left[e, L_{-1}\right]\right)$.

So $\left[y_{0},\left[y_{0}, L\right]\right]=\left[y_{0},\left[y_{0}, L_{0}\right]\right] \subset I_{0}$; i.e., $I_{0}$ is an inner ideal of $L$. 
(ii) Let $0 \neq y_{0} \in I_{0}$ be von Neumann regular. By grading properties, there exists $z_{0} \in L_{0}$ such that $\left[y_{0},\left[y_{0}, z_{0}\right]\right]=-2 y_{0}$. Set $e^{\prime}:=e+y_{0}$. Then, using (1)-(4), it is routine to verify that $e^{\prime}$ is a Jordan element of $L$, i.e., $\operatorname{ad}_{e^{\prime}}^{3}=0$. We also have

$$
\begin{aligned}
{\left[e^{\prime},\left[e^{\prime}, f\right]\right] } & =\left[e+y_{0},\left[e+y_{0}, f\right]\right]=[e,[e, f]]+\left[e,\left[y_{0}, f\right]\right] \\
& +\left[y_{0},[e, f]\right]+\left[y_{0},\left[y_{0}, f\right]\right]=[e,[e, f]]=-2 e,
\end{aligned}
$$

since $\left[y_{0},[e, f]\right]=\left[y_{0}, h\right]=0$ and $\left[y_{0}, f\right]=0$ by $(2)$. We also have

$$
\begin{aligned}
{\left[e^{\prime},\left[e^{\prime}, z_{0}\right]\right] } & =\left[e,\left[e, z_{0}\right]\right]+\left[e,\left[y_{0}, z_{0}\right]\right]+\left[y_{0},\left[e, z_{0}\right]\right] \\
& +\left[y_{0},\left[y_{0}, z_{0}\right]\right]=\left[y_{0},\left[y_{0}, z_{0}\right]\right]=-2 y_{0},
\end{aligned}
$$

since $\left[e,\left[e, z_{0}\right]\right]=0$ by grading properties and $\left[e,\left[y_{0}, z_{0}\right]\right]=\left[y_{0},\left[e, z_{0}\right]\right] \in\left[y_{0}, L_{2}\right]=0$ by (3). Therefore, $e, y_{0} \in\left[e^{\prime}\right]$, and $e^{\prime}=-\frac{1}{2}\left[e^{\prime},\left[e^{\prime}, f+z_{0}\right]\right]$ is von Neumann regular.

(iii) Suppose that $I_{0}=0$. Given $y_{1} \in I_{1}$, we have

$$
\left[y_{1},\left[y_{1}, L_{-2}\right]\right] \subset I \cap L_{0}=I_{0}=0 .
$$

We also have, by grading properties, that

$$
\left[y_{1},\left[y_{1}, L_{i}\right]\right]=0, \text { for } i=1,2 .
$$

Since $I$ is abelian, $y_{1} \in I$ is a Jordan element, so, by 1.5 and (7),

$$
\operatorname{ad}_{\mathrm{ad}_{y_{1}}^{2} x_{0}}^{2} L=\operatorname{ad}_{\mathrm{ad}_{y_{1}}^{2} x_{0}}^{2} L_{-2}=\operatorname{ad}_{y_{1}}^{2} \operatorname{ad}_{x_{0}}^{2} \operatorname{ad}_{y_{1}}^{2} L_{-2}=0,
$$

and hence $\left[y_{1},\left[y_{1}, L_{0}\right]\right]=0$ by nondegeneracy of $L$. Then

$$
\left[y_{1},\left[y_{1}, L\right]\right] \subset\left[y_{1},\left[y_{1}, L_{-1}\right]\right] \subset I \cap L_{1}=I_{1},
$$

and $I_{1}$ is an inner ideal of $L$.

The following result extends one by G. Benkart $[2,1.12]$ for simple nondegenerate Artinian Lie algebras over a field of characteristic 0 or $p>3$.

Lemma 2.2. Let $L$ be a simple nondegenerate Lie algebra containing minimal inner ideals over a ring of scalars $\Phi$ in which 2,3,5,7 are invertible. Then every proper inner ideal of $L$ is abelian.

Proof. Let $0 \neq B$ be a proper inner ideal of $L$. Since minimal inner ideals of $L$ are either abelian or inner-simple ideals [2, 1.12], $L$ contains abelian minimal inner ideals. Then, by the structure theorem of the simple Lie algebras containing abelian minimal inner ideals [4, 5.1] (here the invertibility of 2,3,5,7 in $\Phi$ is needed), there are three possibilities for $L$. If $L$ is finite-dimensional over its centroid, then it is Artinian and hence any proper inner ideal of $L$ is abelian by [2, 1.13]. If $L=[R, R] / Z(R) \cap[R, R]$ (where $R$ is a simple ring with minimal one-sided ideals), then any proper inner ideal of $L$ is of the form $B / Z(R) \cap[R, R]$, where $B$ is a proper inner ideal of $[R, R]$ containing $Z(R) \cap[R, R]$, and we know proper inner ideals of $[R, R]$ are abelian by [1, 3.13]. If $L=[K, K] / Z(R) \cap[K, K]$ (where $K$ is the set of skew elements of a simple ring $R$ with minimal one-sided ideals relative to an involution $*)$, then any proper ideal of $L$ is of the form $B / Z(R) \cap[K, K]$, where $B$ is a proper inner ideal of $[K, K]$. It follows from [1, 4.21] (if the involution is of the first kind) and from [1, 4.26] (if the involution is of the second kind) that $B$ is abelian. 
Proposition 2.3. Let $L$ be a nondegenerate Lie algebra over a ring of scalars $\Phi$ in which 2,3,5,7 are invertible, and let $\operatorname{Soc} L=\bigoplus_{\alpha} M_{\alpha}$ be the decomposition of $\operatorname{Soc} L$ into its simple components. Then every inner ideal $B$ of $\operatorname{Soc} L$ is an inner ideal of $L$. In fact, $B=\bigoplus B_{\alpha}$, where for each index $\alpha$, either $B_{\alpha}=M_{\alpha}$ is a simple component of $\operatorname{Soc} L$ or $B_{\alpha}$ is an abelian inner ideal of $L$ contained in $M_{\alpha}$.

Proof. For each index $\alpha$, denote by $\pi_{\alpha}$ the projection of $\operatorname{Soc} L=\bigoplus M_{\alpha}$ onto $M_{\alpha}$. Then $B_{\alpha}:=\pi_{\alpha}(B)$ is an inner ideal of $M_{\alpha}$. If $B_{\alpha}=M_{\alpha}$, then $M_{\alpha}=$ $\left[M_{\alpha},\left[M_{\alpha}, M_{\alpha}\right]\right]=\left[B,\left[B, M_{\alpha}\right]\right] \subset B$. Suppose then that $B_{\alpha}$ is a proper inner ideal of $M_{\alpha}$. By Lemma 2.2, $B_{\alpha}$ is an abelian inner ideal of $M_{\alpha}$. Let $b_{\alpha}=\pi_{\alpha}(b) \in B_{\alpha}$. Since by [7, 4.2] $b_{\alpha}$ is von Neumann regular, $B_{\alpha}$ is an inner ideal of $L$ by Proposition 1.9. and $B_{\alpha}=\left[B_{\alpha},\left[B_{\alpha}, M_{\alpha}\right]\right]=\left[B,\left[B, M_{\alpha}\right]\right] \subset B$. Hence $B=\bigoplus B_{\alpha}$ and it is an inner ideal of $L$.

Proposition 2.4. Let $L$ be a nondegenerate Lie algebra over a ring of scalars $\Phi$ in which 2,3,5,7 are invertible. If Soc $L$ is an essential ideal of $L$, then every inner ideal $B$ of $L$ containing no nonzero ideals is abelian.

Proof. Suppose first that $L$ is simple. Then $B$ is a proper inner ideal of $L=\operatorname{Soc} L$ and hence $B$ is abelian by Lemma 2.2

Consider now the general case. By [4, 2.5], Soc $L=\bigoplus M_{\alpha}$, where the $M_{\alpha}$ are simple ideals of $L$ coinciding with their socles. Since $M_{\alpha}$ is not contained in $B$, $B \cap M_{\alpha}$ is a proper inner ideal of $M_{\alpha}$. But $M_{\alpha}$ is simple, so $B \cap M_{\alpha}$ is abelian by Lemma 2.2. Let $b \in B, b^{\prime} \in B \cap M_{\alpha}$ and $x \in L$, and set $a:=\left[\left[b, b^{\prime}\right], x\right]$. We have

$$
\begin{aligned}
a & =\left[\left[b, b^{\prime}\right], x\right]=\left[[b, x], b^{\prime}\right]+\left[b,\left[b^{\prime}, x\right]\right] \in B \cap M_{\alpha}, \text { and } \\
{[b, a] } & =\left[b,\left[[b, x], b^{\prime}\right]\right]+\left[b,\left[b,\left[b^{\prime}, x\right]\right]\right] \in B \cap M_{\alpha} .
\end{aligned}
$$

Then $\left[\left[b, b^{\prime}\right],\left[\left[b, b^{\prime}\right], x\right]\right]=\left[\left[b, b^{\prime}\right], a\right]=\left[[b, a], b^{\prime}\right] \in\left[B \cap M_{\alpha}, B \cap M_{\alpha}\right]=0$, and hence $\left[b, b^{\prime}\right]=0$ by nondegeneracy of $L$. Therefore,

$$
\left[B, B \cap M_{\alpha}\right]=0 .
$$

Now let $b, c \in B$ and $y \in M_{\alpha}$, and set $\left.a^{\prime}:=[[b, c], y]\right]$. We have

$$
\begin{aligned}
a^{\prime}= & {[[b, c], y]]=[[b, y], c]+[b,[c, y]] \in B \cap M_{\alpha}, \text { and hence } } \\
& {[[b, c],[[b, c], y]]=\left[[b, c], a^{\prime}\right]=\left[\left[b, a^{\prime}\right], c\right]+\left[b,\left[c, a^{\prime}\right]\right]=0 \text { by }(9) . }
\end{aligned}
$$

It follows from 1.4 that $[B, B] \subset \operatorname{Ann} M_{\alpha}$ for each $M_{\alpha}$; but $\operatorname{Soc} L=\bigoplus M_{\alpha}$, so $[B, B] \subset \operatorname{Ann}(\operatorname{Soc} L)=0$ because $\operatorname{Soc} L$ is an essential ideal, and $B$ is abelian as required.

\section{Lie algebras with essential Artinian socle}

Proposition 3.1. Let $L$ be a nondegenerate Lie algebra with essential Artinian socle over a ring of scalars $\Phi$ in which 2,3,5,7 are invertible. Then $L$ and $\operatorname{Soc} L$ share the same abelian inner ideals, so L satisfies the descending chain condition on abelian inner ideals.

Proof. By Proposition 2.3 every abelian inner ideal of Soc $L$ is an (abelian) inner ideal of $L$. Conversely, let $B$ be an abelian inner ideal of $L$. We claim that $B$ is contained in $\operatorname{Soc} L$. We may assume that $B \neq 0$. Since $\operatorname{Soc} L$ is essential, $B \cap \operatorname{Soc} L \neq 0$ by 1.4. Set $M:=B \cap \operatorname{Soc} L$ and $V:=(M, L / \operatorname{Ker} M)$. Since Soc $L$ is Artinian, $M$ has finite length and the nondegenerate Jordan pair $V$ is 
also Artinian; see [9, Proposition 3.5(v)]. Therefore, $V$ satisfies the ascending chain condition on principal inner ideals and hence there exists an element $e \in M$ generating a principal inner ideal $[e]$ which is maximal in $M$. Moreover, since $V$ is Artinian and nondegenerate, $e$ is von Neumann regular in $V$ [11, 10.17]; i.e., $e$ is part of an idempotent $(e, \bar{f})$ of $V$. Use [9, Proposition 5.4(d)] to extend $(e, \bar{f})$ to an idempotent $(e, f)$ of $L$, and let $L=L_{-2} \oplus L_{-1} \oplus L_{0} \oplus L_{1} \oplus L_{2}$ be its associated Peirce decomposition. By Lemma 2.1(i), $B=B_{0} \oplus B_{1} \oplus B_{2}$, where $B_{i}=B \cap L_{i}$ and both $B_{0}$ and $B_{2}$ are abelian inner ideals of $L$, with $B_{2}$ contained in Soc $L$ because $e \in \operatorname{Soc} L$. Thus, to prove that the whole of $B$ is contained in $\operatorname{Soc} L$, it suffices to show that $B_{0}=0$. Suppose otherwise, i.e. that $B_{0} \neq 0$. By 1.4. $B_{0} \cap \operatorname{Soc} L \neq 0$, so it contains a von Neumann regular element $b_{0}$ [7, 4.2]. Then, by Lemma 2.1(ii), $e^{\prime}=e+b_{0}$ is a von Neumann regular element yielding a principal inner ideal $\left[e^{\prime}\right]$ greater than $[e]$ in $\mathrm{M}$, which is a contradiction. So $B_{0}=0$ and $B=B_{2}+B_{1} \subset[e,[e, L]]+\left[e, L_{-1}\right] \subset \operatorname{Soc} L$, as required.

Now we prove the main result of this paper:

Theorem 3.2. Let $L$ be a nondegenerate Lie algebra with essential socle $S$ over a ring of scalars $\Phi$ in which 2,3,5,7 are invertible. Then the following conditions are equivalent:

(i) $S$ is Artinian.

(ii) $S$ is a complemented Lie algebra and has finitely many ideals.

(iii) $L$ is abelian complemented and has finitely many simple ideals.

Proof. Note first that any of the hypotheses (i), (ii) or (iii) implies that $S$ is a finite direct sum of simple ideals $S_{i}$ coinciding with their socles.

(i) $\Leftrightarrow$ (ii). This is $[8,3.7]$.

(ii) $\Rightarrow$ (iii). Let $B$ be an abelian inner ideal of $L$. By Proposition $3.1 B \subset S$, and since $S$ is abelian complemented [8, 3.7(iii)], $B$ has an abelian complement in $S$; i.e., there exists an abelian inner ideal $C$ of $S$ such that $S=B \oplus \operatorname{Ker}_{S} C=C \oplus \operatorname{Ker}_{S} B$, but $C$ is actually an abelian inner ideal of $L$ by Proposition 2.3. We also note that $B$ has finite length ( $B$ does not contain any infinite properly ascending chain of inner ideals of $L$ ) because $S$ is Artinian [8, 3.7].

We claim that there exists a $\Phi$-submodule $W$ of $L$ contained in $\operatorname{Ker}_{L} B \cap \operatorname{Ker}_{L} C$ such that $L=S \oplus W$. By [9, 3.5(iii)(v)] and [8, 2.7], $V:=(B, C) \cong\left(B, S / \operatorname{Ker}_{S} B\right)$ is a nondegenerate Artinian Jordan pair and hence contains a maximal idempotent $(b, c)$. Note that $b \in[b,[b, S]]=[b,[b, L]]$ and $c \in[c,[c, S]]=[c,[c, L]]$; since, by [1.6. Jordan elements of $S$ are Jordan elements of $L,(b, c)$ is also an idempotent in $L$. Let $L=L_{-2} \oplus L_{-1} \oplus L_{0} \oplus L_{1} \oplus L_{2}$ be the Peirce decomposition of $(b, c)$, as in 1.8 . By Lemma 2.1(i), $B=B_{0} \oplus B_{1} \oplus B_{2}$ and $C=C_{-2} \oplus C_{-1} \oplus C_{0}$. By maximality of $(b, c)$ in $V, B_{0}=C_{0}=0$ (otherwise, taking $0 \neq x \in B_{0}$, which is von Neumann regular because the subquotient $\left(B_{0}, S / \operatorname{Ker}_{S} B_{0}\right)$ is Artinian, and using Lemma 2.1(ii), we would obtain as in Proposition 3.1 that the principal inner ideal generated by $x+b$ is bigger than $[b,[b, S]]$, a contradiction). Hence it follows from Lemma 2.1(iii) that $B_{1}$ and $C_{-1}$ are inner ideals of $L$. Since $L_{-2} \oplus L_{-1} \oplus L_{1} \oplus L_{2} \subset S$, any subspace $W$ of $L_{0}$ such that $L_{0}=\left(S \cap L_{0}\right) \oplus W$ satisfies $L=S \oplus W$. Moreover, $W \subset L_{0} \subset$ $\operatorname{Ker}_{L} B \cap \operatorname{Ker}_{L} C:\left[B,\left[B, L_{0}\right]\right]=\left[B_{2},\left[B_{2}, L_{0}\right]\right]+\left[B_{2},\left[B_{1}, L_{0}\right]\right]+\left[B_{1},\left[B_{2}, L_{0}\right]\right]=0$ (by grading properties), and $\left[B_{1},\left[B_{1}, L_{0}\right]\right] \subset B_{1} \cap L_{2}=0$ (by grading properties and the fact that $B_{1}$ is an inner ideal); similarly, $\left[C,\left[C, L_{0}\right]\right]=0$, which proves the claim. 
Now $B=B_{1} \oplus B_{2} \subset S$ implies $\operatorname{Ker}_{L} C \cap B=\operatorname{Ker}_{S} C \cap B=0$, and by the above, $L=S \oplus W=B \oplus \operatorname{Ker}_{S} C \oplus W=B \oplus \operatorname{Ker}_{L} C$ because $W \subset \operatorname{Ker}_{L} C$; similarly, $L=C \oplus \operatorname{Ker}_{L} B$.

(iii) $\Rightarrow($ i). Since $S$ has finitely many simple components, it suffices to show that any simple component $M$ of the socle is Artinian or equivalently that any proper (and therefore abelian) inner ideal $B$ of $M$ (and therefore also of $L$ ) has finite length. By [9, 3.5(v)(vi)], $V=\left(B, L / \operatorname{Ker}_{L} B\right) \cong\left(B, M / \operatorname{Ker}_{M} B\right)$ is a simple nondegenerate Artinian Jordan pair, so $B$ has finite length, as required.

Remark 3.3. Nondegenerate Artinian Lie algebras have essential Artinian socle; see [4, 2.6] and Proposition 2.3. However, a nondegenerate Lie algebra can have an essential Artinian socle and not be Artinian itself or equivalently (by [8, 3.7]) not be complemented. Consider the infinite dimensional division algebra $\Delta=K((t ; \sigma))$ given by the $\sigma$-twisted Laurent series algebra over a field extension of a base field $F$ by a countable set of indeterminates $K=F\left(\ldots, x_{-1}, x_{0}, x_{1}, \ldots\right), \sigma\left(x_{n}\right)=x_{n+1}$. Following the proof of [3, Example 2], $\Delta$ is infinite dimensional over $[\Delta, \Delta]+Z(\Delta)$ (none of the indeterminates $\left\{x_{i}\right\}$ belong to $[\Delta, \Delta]+Z(\Delta)$ ). Take $L=\Delta^{(-)} / Z(\Delta)$. Then $L$ is strongly prime with inner-simple socle $[\Delta, \Delta]+Z(\Delta) / Z(\Delta)$ by [1,3.15] and [2, 2.2]. But $L$ has an infinite descending chain of inner ideals: take $I_{i}=$ Soc $L+\sum_{k=i}^{\infty} F \cdot e_{k}$, where the cosets $\left\{e_{i}+[\Delta, \Delta]+Z(\Delta)\right\}_{i}$ are linearly independent.

Remark 3.4. One might think that abelian complemented nondegenerate Lie algebras coincide with Lie algebras where complementation holds on their essential socle. However, if $\left\{S_{i}\right\}_{i \in \mathbb{N}}$ is an infinite family of simple nondegenerate Artinian Lie algebras containing abelian inner ideals, then $L=\prod_{i \in \mathbb{N}} S_{i}$ has an essential socle $\bigoplus_{i \in \mathbb{N}} S_{i}$ which is a complemented Lie algebra, but $L$ is not abelian complemented itself. Notice that infinite products of simple nondegenerate Artinian Lie algebras containing abelian inner ideals cannot be abelian complemented: if so, take for every $i \in \mathbb{N}$ a Jordan element $x_{i} \in S_{i}$ and consider $x=\prod_{i \in \mathbb{N}}\left(x_{i}\right) \in L$. Then $B=[x,[x, L]]$ is an abelian inner ideal of $L$ whose subquotient $S=(B, L / \operatorname{Ker} B)$ is a complemented Jordan pair [8, 3.4]. By [13, 5.9] $S$ coincides with its socle, so it satisfies the descending chain condition on principal inner ideals, but there exists an infinite descending chain of inner ideals $B_{n}=\left\{\prod_{i>n}\left(x_{i}\right), L / \operatorname{Ker} B, \prod_{i>n}\left(x_{i}\right)\right\}$ contained in $S$.

\section{REFERENCES}

1. Georgia Benkart, The Lie inner ideal structure of associative rings, J. Algebra 43 (1976), no. 2, 561-584. MR0435149 (55:8110)

2. 232 (1977), 61-81. MR0466242 (57:6122)

3. C. L. Chuang and P. H. Lee, Idempotents in simple rings, J. Algebra 56 (1979), no. 2, 510-515. MR.528592 (80h:16013)

4. Cristina Draper Fontanals, Antonio Fernández López, Esther García, and Miguel Gómez Lozano, The socle of a nondegenerate Lie algebra, J. Algebra 319 (2008), 2372-2394.

5. Antonio Fernández López, Esther García, and Miguel Gómez Lozano, The Jordan socle and finitary Lie algebras, J. Algebra 280 (2004), no. 2, 635-654. MR2089256 (2005i:17007)

6. _ Inner ideals of finitary simple Lie algebras, J. Lie Theory 16 (2006), no. 1, 97-114. MR2196417 (2006m:17020)

7. MR2290916 (2007k:17049)

8. — An Artinian theory for Lie algebras, J. Algebra 319 (2008), 938-951. 
9. Antonio Fernández López, Esther García, Miguel Gómez Lozano, and Erhard Neher, A construction of gradings of Lie algebras, Int. Math. Res. Not. IMRN (2007), no. 16, Art. ID rnm051, 34 pp. MR2353091

10. Nathan Jacobson, Lie algebras, Dover Publications Inc., New York, 1979, republication of the 1962 original. MR559927 (80k:17001)

11. Ottmar Loos, Jordan pairs, Lecture Notes in Mathematics, Vol. 460, Springer-Verlag, Berlin, 1975. MR0444721 (56:3071)

12. MR 1094683 (92a:17050)

13. Ottmar Loos and Erhard Neher, Complementation of inner ideals in Jordan pairs, J. Algebra 166 (1994), no. 2, 255-295. MR1279257 (95f:17028)

14. Erhard Neher, Lie algebras graded by 3-graded root systems and Jordan pairs covered by grids, Amer. J. Math. 118 (1996), no. 2, 439-491. MR1385288 (97a:17029)

15. G. B. Seligman, Modular Lie algebras, Ergebnisse der Mathematik und ihrer Grenzgebiete, Band 40, Springer-Verlag New York, Inc., New York, 1967. MR0245627 (39:6933)

16. E. I. Zelmanov, Lie algebras with algebraic associated representation, Mat. Sb. (N.S.) 121(163) (1983), no. 4, 545-561. MR716113 (84k:17006)

Departamento de Álgebra, Geometría y Topología, Universidad de Málaga, 29071 Málaga, Spain

E-mail address: emalfer@agt.cie.uma.es

Departamento de Matemática Aplicada, Universidad Rey Juan Carlos, 28933 MóstoLES (MADRID), SPAIN

E-mail address: esther.garcia@urjc.es

Departamento de Álgebra, Geometría y Topología, Universidad de Málaga, 29071 Málaga, Spain

E-mail address: magomez@agt.cie.uma.es 